\title{
The impact of AGN on their host galaxies
}

\author{
C. M. Harrison \\ Department of Physics, Durham University, South Road, Durham, DH1 3LE, UK \\ email: c.m.harrison@durham.ac.uk
}

\begin{abstract}
In these proceedings I briefly: (1) review the impact (or "feedback") that active galactic nuclei (AGN) are predicted to have on their host galaxies and larger scale environment, (2) review the observational evidence for or against these predictions and (3) present new results on ionised outflows in AGN. The observational support for the "maintenance mode" of feedback is strong (caveat the details); AGN at the centre of massive halos appear to be regulating the cooling of hot gas, which could in turn control the levels of future star formation (SF) and black hole growth. In contrast, direct observational support for more rapid forms of feedback, which dramatically impact on SF (i.e., the "quasar mode"), remains elusive. From a systematic study of the spectra of $\approx 24000$ AGN we find that extreme ionised gas kinematics are common, and are most prevalent in radio bright $\mathrm{AGN}\left(L_{1.4 \mathrm{GHz}}>10^{23} \mathrm{~W} \mathrm{~Hz}^{-1}\right)$. Follow-up IFU observations have shown that these extreme gas kinematics are extended over kilo-parsec scales. However, the coexistence of high-levels of SF, luminous AGN activity and radio jets raises interesting questions on the primary drivers and impact of these outflows. Galaxy-wide, high-mass outflows are being observed in an increasing number of AGN and are a plausible mechanism for the depletion of gas; however, there is still much work to be done to determine the physical processes that drive these outflows and to measure the level of impact that they have on their host galaxies.
\end{abstract}

Keywords. galaxies: general, galaxies: active, galaxies: evolution, galaxies: jets, galaxies: kinematics and dynamics, ISM: jets and outflows, ISM: kinematics and dynamics

\section{Introduction}

Among the most important discoveries in modern astronomy is that all massive galaxies host a central super-massive black hole $(\mathrm{BH})$. These $\mathrm{BHs}$ primarily grow through mass accretion and become visible as active galactic nuclei (AGN). Surprisingly, observations of galaxies in the local Universe have shown that $\mathrm{BH}$ masses are proportional to that of their host galaxy spheroid (typical mass ratio $\approx 1.4 \times 10^{-3}$; e.g., Kormendy \& Richstone 1995; Magorrian et al. 1998; Temaine et al. 2002; Gültekin et al. 2009) despite a factor of $\approx$ billion difference in their physical size scales. Over the last couple of decades theoretical models of galaxy formation have implemented energetic "feedback" processes between $\mathrm{BH}$ growth and galaxy growth to reproduce this relationship and many other fundamental observable properties of galaxies, intracluster medium (ICM) and the intergalactic medium (IGM). Excellent reviews covering this topic can be found in: Cattaneo et al. (2009); Alexander \& Hickox (2012), McNamara \& Nulsen (2012) and Fabian (2012). In these proceedings, I briefly summarise some of the key results, discuss the most recent research and present new results from a systematic study of ionised outflows in AGN.

\section{Matching models with observations: AGN to the rescue}

AGN are incredible energy sources. During the growth of a $\mathrm{BH}$, huge amounts of energy can be liberated. Assuming that the energy released during mass accretion onto a $\mathrm{BH}$ is $E_{\mathrm{BH}} \approx 0.1 M_{\mathrm{BH}} c^{2}$, to build a $\mathrm{BH}$ with mass $M_{\mathrm{BH}}=10^{8} \mathrm{M}_{\odot}$ would correspond to 
$E_{\mathrm{BH}}=10^{61} \mathrm{erg}$. This total accretion energy is two orders of magnitude higher than the binding energy of the host galaxy spheroid $\left(M_{\mathrm{Sph}} \approx 10^{11} \mathrm{M}_{\odot} ; E_{\mathrm{BE}} \approx 10^{59} \mathrm{erg}\right)$ and can be comparable to, or higher than, the thermal energy of the gas in the dark matter halo (e.g., Bower et al. 2008). If even a small fraction of the accretion energy can couple to the gas over $\approx 0.1-1000 \mathrm{kpc}$, growing $\mathrm{BHs}$ have the potential to regulate their own growth and impact upon the gas in their host galaxies and that in the larger scale environment. Indeed, to successfully reproduce many of the fundamental properties of galaxies, ICM and the IGM models of galaxy formation have found it necessary for AGN to inject some of this energy into the surrounding gas. I discuss some examples of this below.

One of the most famous theoretical results on the impact of AGN comes from semianalytical models, which are unable to reproduce the cut-off at the bright end of the galaxy luminosity function without AGN injecting energy into the halo gas (e.g., Bower et al. 2006; Croton et al. 2006). In these models low accretion rate AGN located in the quasi-hydrostatic halos of massive galaxies (i.e., halo masses $\gtrsim 3 \times 10^{11} h^{-1} \mathrm{M}_{\odot}$ for local galaxies) efficiently suppress the cooling of hot gas through the so-called "maintenancemode" or "radio-mode", resulting in the regulation of future star formation (SF) and $\mathrm{BH}$ growth in the host galaxy. This method of feedback has also been used to explain other observables such as the lack of cold gas in galaxy clusters (e.g., Quilis et al. 2001; Peterson et al. 2003; Gaspari et al. 2011), the colour bi-modality of galaxies and the evolution of cosmic SF density (e.g., Bower et al. 2006; Croton et al. 2006).

The relationship between the X-ray luminosity $\left(L_{\mathrm{X}}\right)$ and X-ray temperature $\left(T_{\mathrm{X}}\right)$ of the ICM within groups and clusters is another key observable for models to reproduce. This is observed to be steeper than expected if gravity was the only source of heating (e.g., Markevitch 1998; Horner 2001; Sun et al. 2009; Stott et al. 2012). AGN are thought to be the most viable source of extra heating (e.g., Valageas \& Silk 1999; Wu, Fabian \& Nulsen 2000) and simulations require that AGN remove some of the low-entropy gas from the centers of halos to reproduce the observed $L_{\mathrm{X}}-T_{\mathrm{X}}$ relationship (e.g., Bower et al. 2008; Puchwein et al. 2008; McCarthy et al. 2010); however, the details of this process are yet to be fully understood (McCarthy et al. 2011; McNamara \& Nulsen 2012).

The "maintenance-mode" of feedback, which has dominated the previous discussion, is thought to be most efficient in the most massive halos, at late times and is associated with BHs with low mass accretion rates (e.g., Churazov et al. 2005; Bower et al. 2006; McCarthy et al. 2011). In contrast, a more rapid/catastrophic form of interaction between AGN and their host galaxies (sometimes named the "quasar mode") is proposed during high accretion states (e.g., Silk \& Rees 1998). To reproduce many fundamental observables of massive galaxies and $\mathrm{BHs}$, simulations that invoke this form of feedback typically require $\approx 5-15 \%$ of the accretion energy to couple to the surrounding gas, expel gas through outflows and consequently suppress or shut down future BH growth and SF (e.g., Benson et al. 2003; DiMatteo et al. 2005; Booth \& Schaye 2010; DeBuhr et al. 2012). Analytical models have also used the idea of galaxy-wide outflows, initially launched by AGN, to explain the BH-mass-galaxy mass relationship $\dagger$ (e.g., Fabian 1999; Murray et al. 2005; King et al. 2011; see review in Alexander \& Hickox 2012). However, there are doubts about whether AGN-driven outflows are globally a sufficient form of feedback (e.g., DeBuhr et al. 2010; 2012) and it is possible that this "quasar mode" is necessary to preheat/expel gas at early times before the "maintenance-mode" takes over at lower redshifts (Gabor et al. 2011; McCarthy et al. 2011).

Finally, AGN-driven outflows (in addition to supernovae) may be required to unbind gas from their host galaxies to fully explain the chemical enrichment of ICM and the IGM (e.g., Borgani et al. 2008; Wiersma et al. 2009; Fabjan et al. 2010) and it has also been

$\dagger$ Another interpretation is this is a natural consequence of repeated mergers (e.g., Peng 2007) 


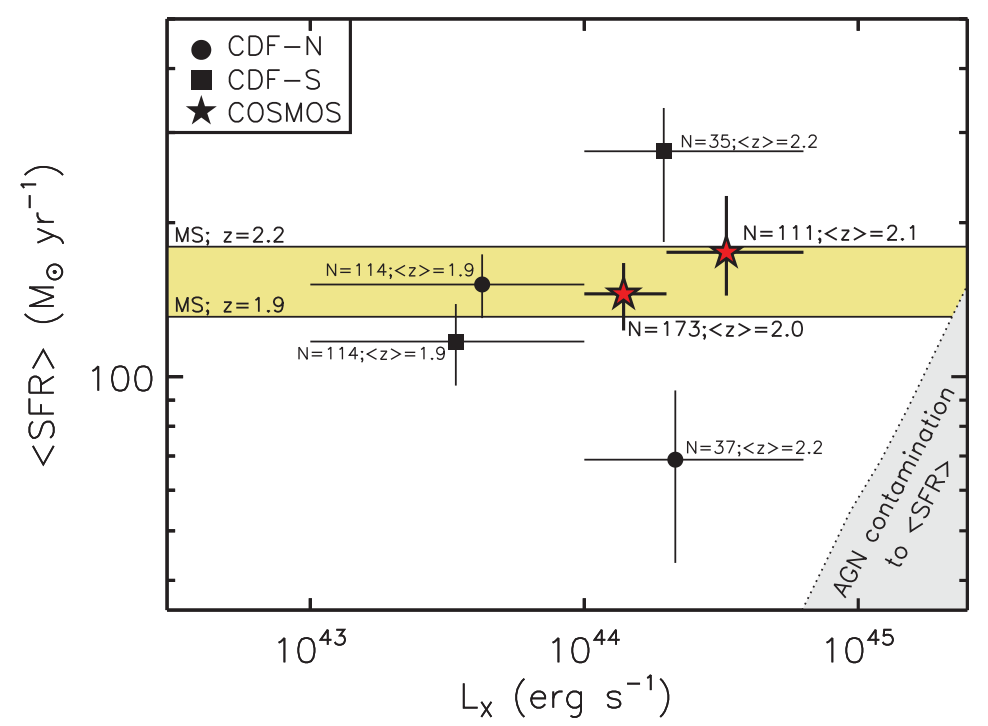

Figure 1. Mean SFR versus X-ray luminosity for AGN in three fields, with the number of sources and mean redshift of each bin indicated. The solid horizontal lines show the typical SFRs for non-AGN star-forming galaxies of comparable masses $\left(\approx 10^{10.9} \mathrm{M}_{\odot}\right)$ at $z=1.9$ and $z=2.2$ (Elbaz et al. 2011). The X-ray luminous AGN have the same mean SFRs as the non-AGN. Variations at the high-luminosity end, that were previously reported in the literature, appear to be due to source statistics and field-to-field variations. Adapted from Harrison et al. (2012a).

proposed that, in some cases, AGN-driven outflows could also cause positive "feedback" by triggering SF episodes through induced pressure of the cold gas (e.g., Nayakshin \& Zubovas 2012; Ishibasi \& Fabian 2012).

Galaxy formation models have proven to be very successful at reproducing global observable properties of galaxies, ICM and the IGM. However, they often rely on diverse, and artificial prescriptions when implementing AGN "feedback" , in particular to how the $\mathrm{BH}$ accretion energy couples to the gas. We must therefore appeal to observations to look for direct evidence that AGN have an impact on their host galaxies and larger scale environment and to constrain the details of how, when and where this impact occurs.

\section{Searching for observational evidence of the impact of AGN}

As already briefly mentioned, there are several pieces of indirect observational evidence that AGN are injecting energy into their larger scale environment inside groups and clusters (e.g., the low rates of cooling; the steep $L_{\mathrm{X}}-T_{\mathrm{X}}$ relationship). However, more direct observational evidence is found through combining X-ray and radio imaging of clusters where cavities in the X-ray emitting gas, indicating the prevention of cooling, are observed spatially co-incident with radio sources (e.g., Boehringer et al. 1993; Carilli et al. 1994; McNamara et al. 2000; Hlavacek-Larrondo et al. 2013). Most of the injected energy is in the form of mechanical energy from jets, which inflate these cavities and is capable of exceeding the X-ray luminosities of their cooling atmospheres (e.g., Bîrzan et al. 2008; Cavagnolo et al. 2010; see McNamara \& Nulsen 2012 for a review). Indeed, the rates of cooling and heating (inferred from X-ray and radio observations respectively) in radio-luminous AGN appear to be in relatively close balance in clusters (e.g., Bîrzan et al. 2004; Dunn \& Fabian 2008) and potentially even in groups and massive elliptical galaxies out to $z \approx 1$ (Best et al. 2006; Smolčić et al. 2009; Danielson et al. 2012; Simpson 
et al. 2013). These observations are examples of the truest form of a "feedback" loop (c.f., the "maintenance mode"; see Section 2) where cooling of gas triggers AGN activity which consequently controls its own fuel supply, in addition to that of SF, by preventing further cooling (also see G. Tremblay's article, these proceedings).

Most of the above focuses on radio AGN in dense environments which are radiatively inefficient (i.e., low-accretion states) and may represent a distinct class of AGN compared to their radiatively efficient counterparts (Hickox et al. 2009; Best \& Heckman 2012). Next I consider observations, particularly at high redshift, that investigate the impact of the radiatively efficient (here-after "luminous") AGN population, which are proposed to be responsible for "quasar mode" feedback (Section 2). Here we are looking for observational signatures that luminous AGN expel gas from their host galaxies, redistribute metals and/or impact on SF (Section 2). Energetic and high-mass outflows do exist in luminous AGN (Section 4); however, observationally it is not yet clear what impact that these AGN have on the formation and evolution of galaxies. A lot of recent observational work has concentrated on investigating the star formation rates (SFRs) in the host galaxies of AGN compared to the overall population (see Alexander \& Hickox et al. 2012; Fabian 2012 for other examples). Subsequent to the surveys carried out by the Herschel satellite, the study of SFRs of AGN has increased greatly, largely because the far-infrared wavelengths observed $(\lambda=70-500 \mu \mathrm{m})$ arguably provide the most reliable SFR measurements in luminous AGN (e.g., Mullaney et al. 2012). Herschel results show that, on average, the SFRs of X-ray selected AGN with moderate luminosities (i.e., $L_{\mathrm{X}}=10^{42}-10^{44} \mathrm{erg}$ ) are consistent with non-AGN star-forming galaxies of the same mass and redshift (e.g., Shao et al. 2010; Mullaney et al. 2012) in agreement with other work (e.g., Xue et al. 2010; Lutz et al. 2010; Mainieri et al. 2011). However, the mean SFRs of the most luminous X-ray $\operatorname{AGN}\left(L_{\mathrm{X}}>10^{44} \mathrm{erg} \mathrm{s}^{-1}\right)$ is a more controversial topic pre- and post-Herschel, with claims of both enhanced and suppressed SFRs compared to the overall population (e.g., Lutz et al. 2010; Rovilos et al. 2012; Page et al. 2012). However, a major factor behind these different conclusions is poor source statistics and/or field-to-field variations (Harrison et al. 2012a; see Fig. 1). When looking at large samples of X-ray AGN the mean SFRs are consistent with the overall star-forming population out to $L_{\mathrm{x}} \approx 10^{45} \mathrm{erg} \mathrm{s}^{-1}$, at least over the redshift range $z=1-3$ (see Fig. 1; see Rosario et al. 2012). Taken at face-value, these results might appear to suggest that luminous AGN have no impact on $\mathrm{SF}$; however, there are many factors to consider. For example, depending on the relative timescales and variability of X-ray luminous AGN activity compared to SF episodes (see Hickox et al. 2013) any subtle signatures of SFR suppression or enhancement in AGN could be very challenging to detect using these methods (Harrison et al. 2012a).

Finally, directly conflicting studies of different classes of AGN (e.g., radio luminous, X-ray luminous) have recently arisen in the literature, arguing that each class of AGN are capable of suppressing, enhancing, or having no impact on SF (e.g., Kalfountzou et al. 2012; Zinn et al. 2013; Karouzos et al. 2013; Feltre et al. 2013; Rosario et al. 2013). There are potentially many explanations for this. Sample selection is likely to be a key factor, for example, constructing samples based on shallow radio or infrared surveys will only identify objects with very high SFRs. Also, the various approaches for selecting AGN at different wavelengths (i.e., X-rays, optical, mid-infrared or radio), all have different issues of contamination (from non-AGN) and completeness and may even result in selecting AGN of different masses, evolutionary stages or environments (Hickox et al. 2009; Goulding et al. 2013). We must first understand the many observational biases and obtain SFR distributions, down to reasonable limits, for complete samples before we can draw firm conclusions on the impact of luminous AGN on SF. 

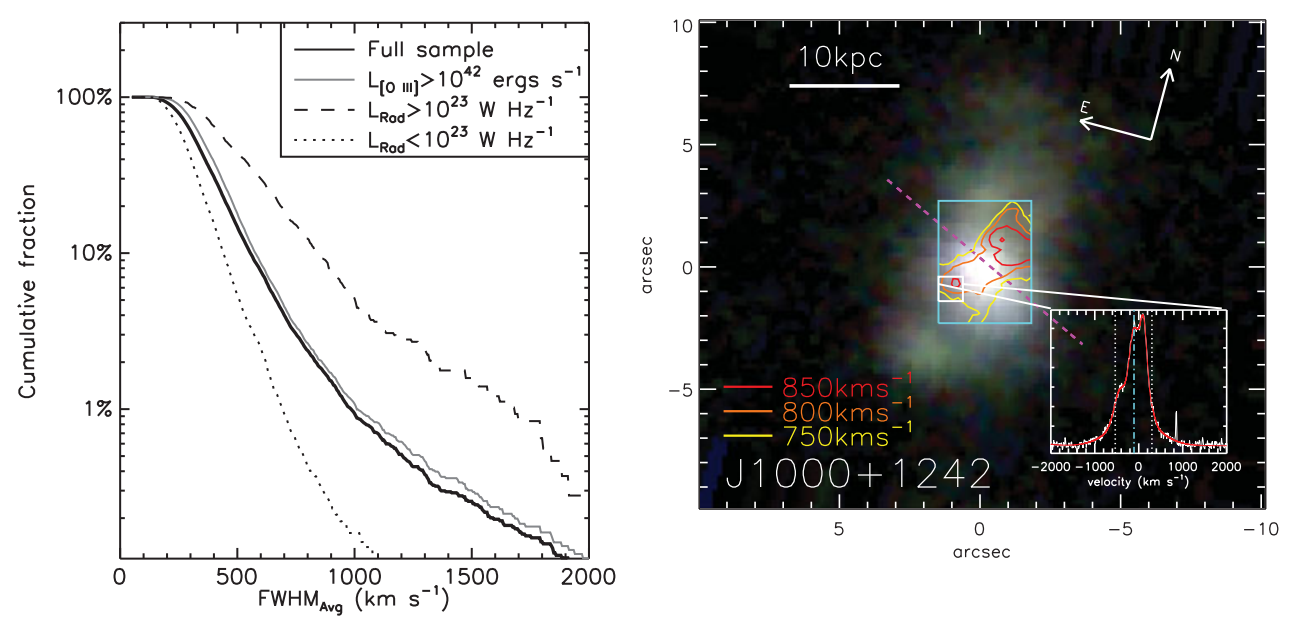

Figure 2. Left: The fraction of $z<0.4 \mathrm{AGN}$ with $\left[\mathrm{O}\right.$ III] $\mathrm{FWHM}_{\mathrm{Avg}}$ (weighted average of broad and narrow components) greater than the given values. Values of $\mathrm{FWHM}_{\mathrm{Avg}}>1000 \mathrm{~km} \mathrm{~s}^{1}$ are $\approx 5$ times more prevalent among AGN with $\mathrm{L}_{1.4 \mathrm{GHz}}>10^{23} \mathrm{~W} \mathrm{~Hz}^{1}$ (Mullaney et al. 2013). Right: The SDSS image of an example AGN from our follow-up study of the Mullaney et al. (2013) parent sample Harrison et al. (2014). Our IFU data (contours) reveal a high-velocity bi-polar ionised outflow, perpendicular to the galactic kinematic major axis (dashed line). The inset shows an [O III] emission-line profile from the indicated region. Kiloparsec-scale extreme ionised gas kinematics are observed in all of the sixteen AGN with IFU data Harrison et al. (2014).

\section{Galaxy-wide outflows: a "lever-arm" for AGN to impact on their host galaxies}

AGN-driven, galaxy-wide outflows are a key prediction of many models (Section 2). AGN outflows are known to be common on $\lesssim 1$ pc scales since very high-velocity winds (up to $\approx 0.1 \mathrm{c}$ ) are observed in a large fraction of AGN and may even be ubiquitous (e.g., Pounds et al. 2003; Ganguly \& Brotherton 2008 Tombesi et al. 2010; see Fabian 2012 for review). However, while significant, these studies provide little direct insight into what effect these outflows have on the gas and SF over galactic scales. This requires spatiallyresolved kinematic measurements of ionised gas, molecular gas and atomic gas. Here I concentrate on ionised outflows which currently provide the easiest means to perform statistical studies (for observations in other phases see S. Veilleux, these proceedings). Broad $\left(>500-1000 \mathrm{~km}^{-1}\right)$, high-velocity and spatially extended [O III $] 5007$ emission is one key diagnostic that has revealed galaxy-wide ionised outflows in integral field spectroscopy (IFU data) of low and high redshift AGN (e.g., Nesvadba et al. 2008; Ruke \& Veilleux 2011; Harrison et al. 2012b; Liu et al. 2013). These IFU observations have demonstrated that galaxy-wide ionised outflows exist and have the potential to drive gas out of their host galaxies; however, they are typically of small and inhomogeneous samples of AGN. Key questions that arises from these studies are: "how representative are the objects of the overall population?" and "where are ionised outflows most preferentially found?".

To address the questions above we performed emission-line de-composition (fitting broad and narrow components) on $\approx 24000 z<0.4$ AGN from the SDSS survey (Mullaney et al. 2013). We consequently measured the prevalence of ionised outflow features in the overall AGN population and developed a well constrained parent sample for the basis for detailed follow-up observations. After accounting for the known correlation between bolometric luminosity $\left(L_{\mathrm{AGN}}\right)$ and radio luminosity $\left(L_{1.4 \mathrm{GHz}}\right)$, we found that the most extreme line widths are preferentially found in radio bright systems $\left(L_{1.4 \mathrm{GHz}}>10^{23} \mathrm{~W} \mathrm{~Hz}^{-1}\right.$; see Fig. 2) whilst no clear trends are observed with 
$L_{\mathrm{AGN}}$ or Eddington ratio. Follow-up IFU observations of sixteen of the luminous AGN $\left(L_{[\mathrm{O}}\right.$ III $]>10^{41.7} \mathrm{erg} \mathrm{s}^{-1}$; Harrison et al. $\left.(2014)\right)$ have shown that the high-velocity features, identified in Mullaney et al. (2013), are extended over $\gtrsim(6-16) \mathrm{kpc}$ in all cases, and show a range of morphology and structure (e.g., see Fig. 2). We measure outflow properties, SFRs and AGN luminosities and search for evidence of radio jets in the sample. The implied mass outflow rates are comparable to the host galaxy SFRs [i.e., $\lesssim(7-100) \mathrm{M}_{\odot} \mathrm{yr}^{-1}$ ] and the energy injection rates are high $\left(\approx 10^{41-43} \mathrm{erg} \mathrm{s}^{-1}\right)$ in broad agreement with predictions of the "quasar mode" of feedback (Section 2). The observed AGN have radio luminosities of $L_{1.4 \mathrm{GHz}} \gtrsim 10^{23} \mathrm{~W} \mathrm{~Hz}^{-1}$; however, their radio emission is from a mixture of SF and AGN activity, raising the interesting question: which physical process is responsible for the most extreme ionised gas kinematics? Full results and discussion of the IFU sample will soon be presented in Harrison et al. (2014).

As a final note, it is worth remembering that the existence of galaxy-wide outflows is not direct proof that they have a long-term impact on their host galaxies. Even the most massive outflows travelling at the galaxy escape velocity may stall in the galaxy halo, re-collapse and cool at later times (along with new fuel supplies), resulting in the re-ignition of SF and $\mathrm{BH}$ growth (e.g., Harrison et al. 2012b; Lagos et al. 2008; McCarthy et al. 2011; Gabor et al. 2011; Rosas-Guevara et al. 2013). We have made great progress in identifying and characterising outflows in AGN; however, there is crucial work still to be done to measure the impact that galaxy-wide outflows have on their host galaxies.

\section{References}

Alexander D. M., Hickox R. C., 2012, New Astron. Revs, 56, 93

Benson A. J., et al. 2003, ApJ, 599, 38

Best P. N. \& Heckman T. M., 2012, MNRAS, 421, 1569

Best P. N., et al. 2006, MNRAS, 368, L67

Bîrzan L., et al. 2008, ApJ, 686, 859

Bîrzan L., et al. 2004, ApJ, 607, 800

Boehringer H., et al. 1993, MNRAS, 264, L25

Booth C. M. \& Schaye J., 2010, MNRAS, 405, L1

Borgani S., et al. 2008, Space Sci. Revs, 134, 379

Bower R. G., et al. 2006, MNRAS, 370, 645

Bower R. G., McCarthy I. G., \& Benson A. J., 2008, MNRAS, 390, 1399

Carilli C. L., Perley R. A., \& Harris D. E., 1994, MNRAS, 270, 173

Cattaneo A., et al. 2009, Nature, 460, 213

Cavagnolo K. W., et al. 2010, ApJ, 720, 1066

Churazov E., et al. 2005, MNRAS, 363, L91

Croton D. J., et al. 2006, MNRAS, 365, 11

Danielson A. L. R., et al. 2012, MNRAS, 422, 494

Debuhr J., Quataert E., \& Ma C.-P., 2012, MNRAS, 420, 2221

Debuhr J., et al. 2010, MNRAS, 406, L55

Di Matteo T., Springel V., \& Hernquist L., 2005, Nature, 433, 604

Dunn R. J. H. \& Fabian A. C., 2008, MNRAS, 385, 757

Elbaz D., et al. 2011, A\&A, 533, A119

Fabian A. C. 1999, MNRAS, 308, L39

Fabian A. C. 2012, AAESAR, 50, 455

Fabjan D., et al. 2010, MNRAS, 401, 1670

Feltre A., et al. 2013, MNRAS, 434, 2426

Gabor J. M., Davé R., Oppenheimer B. D., \& Finlator K., 2011, MNRAS, 417, 2676

Ganguly R. \& Brotherton M. S., 2008, ApJ, 672, 102

Gaspari M., et al. 2011, MNRAS, 411, 349 
Goulding A. D., et al. 2013, arXiv:1310.8298

Gültekin K., et al. 2009, ApJ, 698, 198

Harrison C. M., et al. 2012a, ApJ (Letters), 760, L15

Harrison C. M., et al. 2012b, MNRAS, 426, 1073

Harrison C. M., et al. 2014, MNRAS, 441, 3306

Hickox R. C., et al. 2009, ApJ, 696, 891

Hickox R. C., et al. 2013, arXiv:1306.3218

Hlavacek-Larrondo J., et al. 2013, ApJ, 777, 163

Horner D. J., 2001, PhD thesis, University of Maryland College Park

Ishibashi W. \& Fabian A. C., 2012, MNRAS, 427, 2998

Kalfountzou E., et al. 2012, MNRAS, 427, 2401

Karouzos M., et al. 2013, arXiv:1309.7353

King A. R., Zubovas K., \& Power C., 2011, MNRAS, 415, L6

Kormendy J. \& Richstone D., 1995, AAESAR, 33, 581

Lagos C. D. P., Cora S. A., Padilla N. D., 2008, MNRAS, 388, 587

Liu G., et al. 2013, MNRAS, 436, 2576

Lutz D., et al. 2010, ApJ, 712, 1287

Magorrian J., et al. 1998, AJ, 115, 2285

Mainieri V., et al. 2011, A\&BA, 535, A80

Markevitch M., 1998, ApJ, 504, 27

McCarthy I. G., et al. 2011, MNRAS, 412, 1965

McCarthy I. G., et al. 2010, MNRAS, 406, 822

McNamara B. R. \& Nulsen P. E. J., 2012, New Journal of Physics, 14, 055023

McNamara B. R., et al. 2000, ApJ (Letters), 534, L135

Mullaney J. R., et al. 2013, MNRAS, 433, 622

Mullaney J. R., et al. 2012, MNRAS, 419, 95

Murray N., Quataert E., \& Thompson T. A., 2005, ApJ, 618, 569

Nayakshin S. \& Zubovas K., 2012, MNRAS, 427, 372

Nesvadba N. P. H., et al. 2008, A\& A, 491, 407

Page M. J., et al. 2012, Nature, 485, 213

Peng C. Y., 2007, ApJ, 671, 1098

Peterson J. R., et al. 2003, ApJ, 590, 207

Pounds K. A., et al. 2003, MNRAS, 345, 705

Puchwein E., Sijacki D., \& Springel V., 2008, ApJ (Letters), 687, L53

Quilis V., Bower R. G., \& Balogh M. L., 2001, MNRAS, 328, 1091

Rosas-Guevara Y. M., et al. 2013, arXiv:1312.0598

Rosario D. J., et al. 2012, A\&A A, 545, A45

Rosario D. J., et al. 2013, arXiv:1310.1922

Rovilos E., et al. 2012, A\&GA, 546, A58

Rupke D. S. N. \& Veilleux S., 2011, ApJ (Letters), 729, L27

Shao L., et al. 2010, A\& A, 518, L26

Silk J. \& Rees M. J., 1998, A\&AA, 331, L1

Simpson C., et al. 2013, MNRAS, 433, 2647

Smolčić V., et al. 2009, ApJ, 696, 24

Stott J. P., et al. 2012, MNRAS, 422, 2213

Sun M., et al. 2009, ApJ, 693, 1142

Tombesi F., et al. 2010, A\&BA, 521, A57

Tremaine S., et al. 2002, ApJ, 574, 740

Valageas P., Silk J., 1999, A\& $A$, 350, 725

Wiersma R. P. C., et al. 2009, MNRAS, 399, 574

Wu K. K. S., Fabian A. C., \& Nulsen P. E. J., 2000, MNRAS, 318, 889

Xue Y. Q., et al. 2010, ApJ, 720, 368

Zinn P.-C., et al. 2013, ApJ, 774, 66 